\title{
Avaliação de biópsias hepáticas em cães: utilização de critérios de análise histopatológica*
}

\author{
Evaluation of hepatic biopsy in dogs: use of histopathological \\ criteria analysis*
}

\author{
Raimundo Alberto Tostes, ${ }^{\star \star}$ Enio Pedone Bandarra, ${ }^{\star \star \star}$ Veridiana Maria Brianezi Dignani de Moura ${ }^{\star \star \star \star}$
}

\begin{abstract}
Resumo
O objetivo do presente trabalho foi avaliar histologicamente 60 casos de doença hepática em cães, aplicando-se critérios de análise histopatológica pré-definidos. As amostras foram obtidas por meio de biópsia percutânea transabdominal com aguIha tipo cortante. Os resultados mostraram um predomínio das alterações em fase aguda (65\%) dos casos e as demais alterações variando de hepatites crônicas a infiltração neoplásica metastática. São descritos os critérios usados e o predomí-
\end{abstract}

Palavras-chave: biópsia hepática; critérios histopatológicos; fígado; cães.

\begin{abstract}
The purpose of the present work was to evaluate histologically 60 cases of hepatic disease in dogs being applied predefined histopathological analysis criteria. The samples were obtained through transabdominal percutaneous biopsy with cutting needle. The results showed a prevalence of the alterations in acute phase $(65 \%)$ and the other alterations varying from chronic hepatitis to neoplasic methastatic infiltration. The used criteria and the prevalence of the lesion degrees are described.
\end{abstract}

Keywords: hepatic biopsy; histopathologic criteria; liver; dogs.

\section{Introdução}

As hepatites nas formas agudas e, principalmente, nas formas crônicas constituem um grupo de doenças de alta prevalência na população canina. Formas crônicas insidiosas e debilitantes exibem características morfológicas peculiares. Os padrões morfológicos de doença hepática em cães evoluiram significativamente quanto à etiopatogenia e nomenclatura, criando uma identidade própria que se diferenciou do modelo humano, antes usado para explicar diversas entidades de doença hepática. Entretanto, a definição de muitos aspectos de doenças hepáticas, especialmente as crônicas, permanece controversa (Dillon, 1985; Dial, 1995).

Os diversos aspectos morfológicos observados na doença hepática incluem, principalmente, a atividade necro-inflamatória, graus de degeneração, fibrose e colestase. LeveilleWebster e Center (1995) destacam que, quando as alterações inflamatórias estão presentes na biópsia hepática, é provável que as células inflamatórias, seus mediadores e citocinas locais liberadas estejam contribuindo para a ocorrência de necrose e início de fibrogênese. A avaliação dos graus de fibrose, valiosa para caracterizar os estágios inter- mediários entre a hepatite crônica e a cirrose em cães, não é marcante, a despeito da etiopatogênese bem definida das hepatites crônicas virais humanas. Thornburg et al. (1983d) afirmam que nos estágios intermediários surgem condições propícias para a proliferação de fibroblastos, a produção de colágeno e a regeneração de hepatócitos. Essas alterações desencadeariam a conformação clássica da cirrose, com pontes de fibrose completas e nódulos de regeneração. Entretanto, a etiopatogênese da cirrose canina, na visão de Thornburg et al. (1983a, 1983b, 1983c, 1983d), limita-se a uma condição pós-necrose; Leveille-Webster e Center (1995), Sevelius (1995), e Sevelius e Jönsson (1995) discutem uma evolução para a cirrose canina mais abrangente, em que efeitos modulatórios da resposta imune desenvolvem um papel essencial, surgindo, em um segundo plano, os fenômenos produtivos, como a fibrogênese. Este último aspecto parece particularmente mais aceitável, visto que nem todas as formas de cirrose e condições fibróticas do fígado pré-cirróticas apresentam características que permitam discernir sobre a sua possivel atividade necro-inflamatória; isto é ainda mais interessante, considerando-se o fato de que nem todas as formas de cirrose exibem atividade inflamatória (Twedt, 1985).

\footnotetext{
* Projeto financiado pela FAPESP (Proc. 95/6577-8).

** Professor Assistente de Anatomia Patológica - Hospital Veterinário - UNOESTE. Rod. Raposo Tavares Km 572, CEP 19001-970, Presidente
Prudente, SP. E-mail: tost@ uol.com.br. Autor para correspondência.

*** Prof. Adj. Dr. Serviço de Patologia do Departamento de Clínica Veterinária - FMVZ - UNESP - Botucatu/SP.

**** Doutoranda do Serviço de Patologia do Depto de Clínica Veterinária - FMVZ - UNESP.
} 
A mera referência de hepatite crônica não colabora com o clínico na evolução para um procedimento terapêutico efetivo. Por extensão, o simplismo diagnóstico, no que se refere às hepatites, apenas referencia uma apresentação morfológica, dissonante daquilo que o clínico espera ouvir, ou seja, uma perspectiva do comportamento biológico da atividade inflamatória em questão. Sherlock (1989) expõe que a classificação exata do tipo de hepatite crônica é claramente importante por causa das implicações prognósticas. A utilização de critérios de análise histopatológica na doença hepática canina implica diretamente a determinação de fatores prognósticos. A instituição destes critérios substancia as classificações morfológicas das hepatites para, à luz da correlação anátomoclínica, entendê-las e interpretá-las, mormente quando o diagnóstico é descritivo. Alguns achados histopatológicos como necrose, inflamação e fibrose respondem freqüentemente às indagações quanto à progressão da doença no seu curso natural ou como resposta do parênquima hepático à terapia instituída.

\section{Material e métodos}

Foram utilizados 60 animais, encaminhados ao Serviço de Patologia da FMVZ, sem distinção de sexo, raça ou idade, com indicação clínica e/ou laboratorial de doença hepática.

Os animais foram submetidos à biópsia percutânea transabdominal por agulha tipo Tru-Cut ${ }^{\circledR}$. As amostras colhidas foram fixadas em solução de formalina neutra a $10 \%$. Processadas para inclusão em parafina e submetidas a cortes histológicos de $5 \mu$. As lâminas foram, então, submetidas a um painel de colorações que incluiu a Hematoxilina-Eosina, Tricrômio de Massom, Warthy-Starry, Sirius Red e P.A.S., com e sem reação com diastase.

Os critérios de análise histopatológica foram definidos assim:

A característica do processo quanto à duração, se agudo ou crônico. Os achados morfológicos relacionados: inflamação, degeneração, necrose, fibrose e colestase. O tipo de degeneração: vacuolar, esteatose (macro e/ou microvacuolar) e glicogênica. A graduação do padrão de inflamação: grau 1(portal): infiltrado inflamatório confinado ao espaço porta e/ou lobular focal; grau 2 (peri-portal discreto) infiltrado inflamatório no espaço porta e parênquima peri-portal (zona 1); grau 3 (peri-portal grave): infiltrado inflamatório nos espaços porta e parênquima peri-portal (zona 1); grau 4 (lobular): infiltrado inflamatório dentro do lóbulo, com presença de lesões hepatocelulares (degeneração e regeneração), hepatócitos necróticos, anisonucleose, atividade mitótica e edema. A natureza do infiltrado inflamatório, procurando-se verificar: polimorfonucleares, linfócitos, plasmócitos e macrófagos. O tipo c'e necrose, se lítica ou isquêmica. A graduação do processo necro-inflamatório: grau 1: atividade mínima, infiltrado inflamatório discreto, necrose lobular focal, grau 2: atividade discreta, infiltrado inflamatório discreto, necrose lobular escassa, grau 3: atividade moderada, infiltrado inflamatório moderado, necrose lobular "salpicada"; grau 4: atividade grave; infiltrado inflamatório marcante, áreas de necrose confluentes resultando em pontes. Nesta graduação, foi observada também a integridade de placa limitante. A graduação de fibrose foi analisada desta forma: grau 1: expansão do espaço porta por fibrose discreta; grau 2: septos de fibrose porto-portais raros; grau 3: septos porto-portais e com veias centrais com distorção mínima da arquitetura, sem formar nódulos de regeneração; grau 4: cirrose, pontes fibrosas completas e nódulos de regeneração. As alterações biliares foram assim graduadas: grau 1: colestase canalicular discreta a moderada; grau 2: colestase canalicular discreta a moderada, com proliferação de células de Küpffer; grau 3: colestase acompanhada de proliferação ductal focal; grau 4: colestase acompanhada de proliferação ductal difusa e fibrose portal. Outras alterações incluíram a presença de granulomas, pigmentos e estrutura vascular. A caracterização de neoplasias foi feita determinando-se a origem (primária ou metastática), a histogênese (mesenquimal ou epitelial) e o comportamento biológico (benigna ou maligna).

A nomenclatura utilizada na definição dos diagnósticos histopatológicos (biópsia e necrópsia) foi baseada em Bianchi (1983), Kelly (1993) e Jones et al. (1997).

\section{Resultados}

Em $65 \%$ dos casos as lesões encontradas foram caracterizadas como sendo de manifestação aguda, variando de alterações degenerativas a necro-inflamatórias. As lesões crônicas, incluindo alterações inflamatórias, fibróticas e neoplásicas, alcançaram $33 \%$. Apenas $2 \%$ dos casos não exibiam qualquer alteração patológica, sendo caracterizados como normais. A maioria das lesões era de natureza primária do fígado. A distribuição das alterações encontradas é observada no Gráfico 1. As alterações degenerativas foram diagnosticadas em $20 \%$ dos casos. Dentro do presente trabalho, a ampla maioria dos casos que apresentaram algum tipo de degeneração hepática incluía a administração prévia de analgésicos não-esteróides. As hepatites agudas foram diagnosticadas em $33 \%$ dos casos.

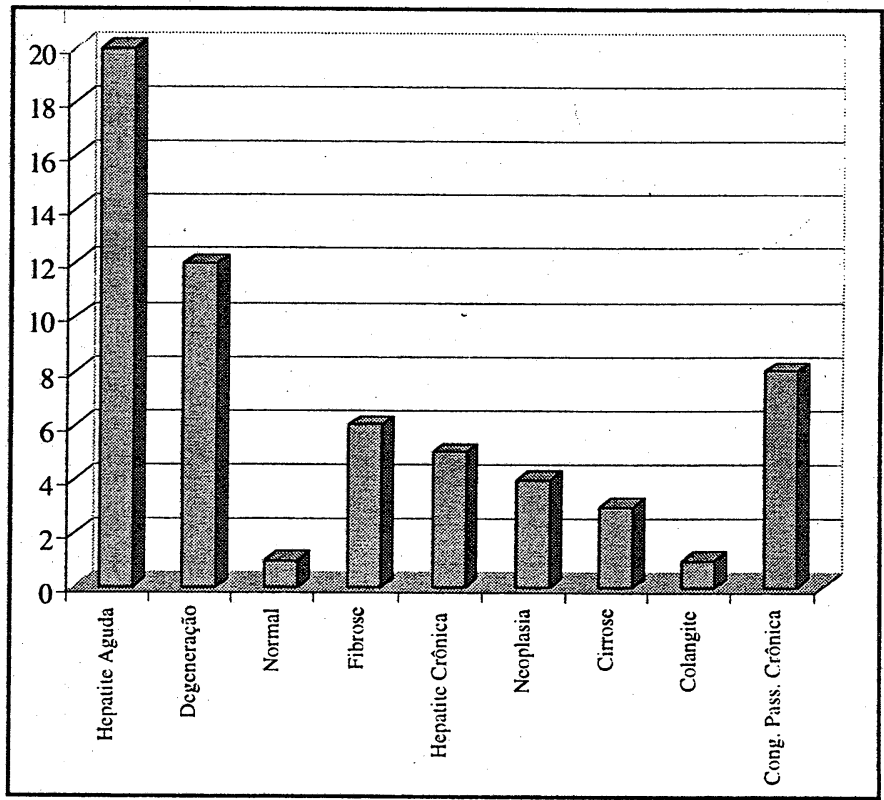

Gráfico 1: Distribuição das lesões observadas ña análise histopatológica de amostras hepáticas. 
Como resultado das variáveis observadas no exame histopatológico, verificou-se um predomínio do padrão lobular, com apresentação variando de focal a multifocal. A natureza do infiltrado inflamatório acompanhou o padrão observado nas amostras de biópsia: polimorfonucleares na fase aguda e mononucleares na crônica, eventualmente caracterizando-se a presença de plasmócitos. A atividade necro-inflamatória mormente era lítica em grau 1, mas eventualmente atingindo até grau 3. As formas graves de hepatite atingiram grau 4. Os graus de fibrose situaram-se de 1 a 3 e os graus de alterações biliares de 1 a 2 . As alterações degenerativas predominaram na forma de degeneração hidrópica e esteatose macrovacuolar. A distribuição dos graus de atividade necro-inflamatória, fibrose e alterações biliares é observada na Tabela 1.

Tabela 1: Distribuição dos graus de lesão observados histologicamente em 60 casos de doença hepática canina.

\begin{tabular}{c|c|c|c}
\hline Grau & $\begin{array}{c}\text { Graduação da atividade } \\
\text { necro-inflamatória }\end{array}$ & $\begin{array}{c}\text { Grau de } \\
\text { fibrose }\end{array}$ & $\begin{array}{c}\text { Grau de } \\
\text { alteracaão biliar }\end{array}$ \\
\hline Grau 1 & $19(31,7 \%)$ & $10(16,7 \%)$ & $08(13,3)$ \\
\hline Grau2 & $03(5 \%)$ & $09(15 \%)$ & $03(5 \%)$ \\
\hline Grau 3 & $06(10 \%)$ & $07(11,7 \%)$ & $01(1,7 \%)$ \\
\hline Grau 4 & $05(8,3 \%)$ & $03(5 \%)$ & $02(3,3 \%)$ \\
\hline
\end{tabular}

O tipo de colágeno encontrado nos processos fibróticos e cirróticos foram predominantemente os tipos I e III, evidenciados na coloração pelo Sirius Red, vișto ao microscópio óptico por meio de luz polarizada. Nesta forma de visualização, as fibras colágenas aparecem birrefrigentes, as de tipo $1 \mathrm{em}$ tonalidade amarelada ou levemente avermelhada, as do tipo II em tonalidades variadas e as do tipo III em verde.

As Figuras 1 e 2 ilustram dois espécimes de biópsia, os quais exibem um número de tratos portais superior a quatro e em que se observam graus variáveis de fibrose.

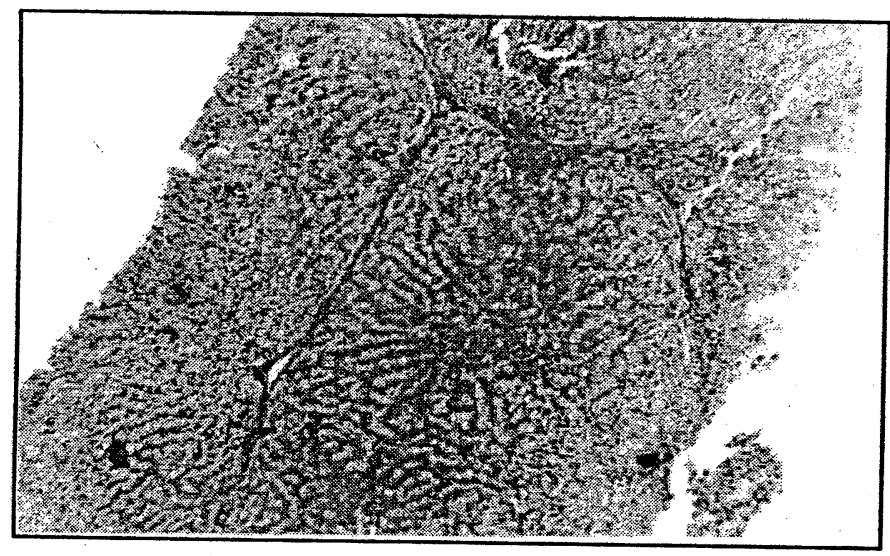

Figura 1: Amostra na qual se observam densos depósitos de fibrose formando pontes portáteis e com a presença de nódulos de regeneração (Tricrômico de Massom, 10X)

\section{Discussão}

Os critérios de análise histopatológica mostraram-se aplicáveis a todas as categorias de lesões observadas. As alterações degenerativas observadas reforçam o fato de que elas ocorram de forma transitória no fígado, exceto na persistên-

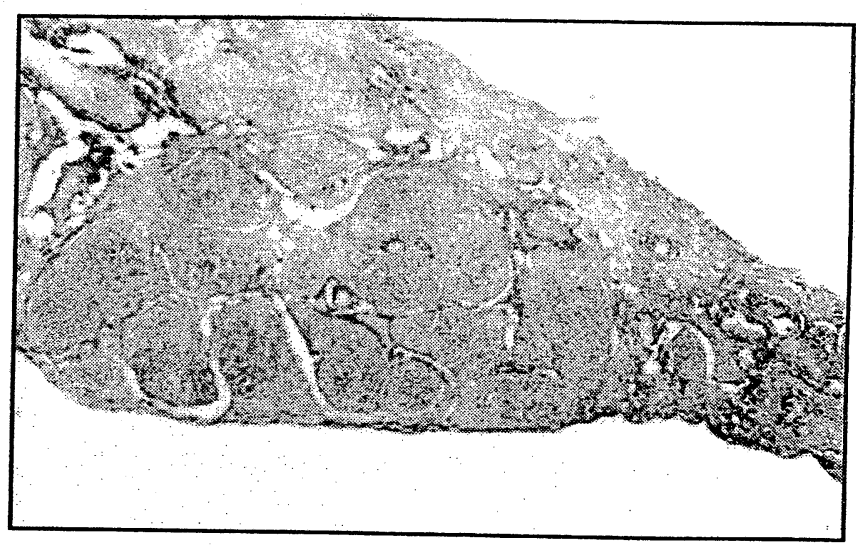

Figura 2: Ponte fibrótica porto-portal com presença de esteatose macro e microvacuolar observada no parênquima (Tricrômico de Massom, 40X).

cia do agente agressor, o que, na maioria das vezes, referese a drogas (Lees et al., 1991; Mckellar et al., 1991; Pessayre, 1995). Os quadros necro-inflamatórios, na forma das hepatites agudas, não diferem substancialmente entre si. Mas, o principal aspecto relacionado a um episódio agudo de hepatite é a sua duração e intensidade, que serão determinantes na progressão da doença e na sobrevida do animal. O mecanismo da transição de uma hepatite aguda para uma hepatite crônica ainda é obscuro na sua maior parte (Center, 1995 e 1996).

As hepatites crônicas espontâneas ainda não têm uma etiologia definida em cães. Contudo, alguns pesquisadores têm enfatizado a correlação entre as formas crônicas de hepatite canina e humana (Sarli, 1992; Adamus et al., 1997). A hepatite crônica canina freqüentemente é idiopática; entretanto, algumas causas específicas possuem predisposição racial: como as hepatites crônicas nos Dobermann Pinschers (Hardy, 1985), Cocker Spaniels Americanos e Cocker Spaniels Escoceses (Andersson e Sevelius, 1991), Bedlington Terriers (Teske et al., 1992) e West Highland White Terriers (Thornburg et al., 1996). Outras formas de hepatite crônica canina têm origem metabólica, como a acumulação hepática de $\alpha$-1-antitripsina (Sevelius et al., 1994), induzida por drogas (Rutgers e Haywood, 1988), infecciosa, como infecções seguidas por Leptospira spp (Bishop et al., 1979, Adamus et al., 1997), hepatite persistente pelo vírus da hepatite de células acidofílicas (Jarrett et al., 1987), pelo Adenovírus Canino tipo 1 (Rakish et al., 1986), ou possivelmente auto-imune, como sugerem Andersson e Sevelius (1992) e Weiss et al. (1995), que encontraram auto-anticorpos circulantes em cães com doença hepática crônica. Poitout et al. (1997) também sustentam que a modulação da resposta imune esteja envolvida em alguns casos de hepatite crônica, as quais Strombeck et al. (1988) mostraram ser responsivas à corticoterapia. No presente estudo, as hepatites crônicas corresponderam a $8 \%$ dos casos. Quatro casos, diagnosticados como hepatite crônica, apresentavam nuanças que permitiam classificá-las de acordo com a nomenclatura de Sevelius et al. (1994) ou Fuentealba (1997) ou mesmo extrapolar a nomenclatura médica de Ishak (1994) e Ludwig (1995) para uso veterinário. Contudo, a essência não se refere à nomenclatura, mas aos elementos envolvidos na lesão. Em um dos casos, os achados de destruição da placa limitante, mais a presença de infiltrado inflamatório linfoplasmocitário são referências morfológicas seguras de uma hepatite crônica ativa, mas 
como as referências clínicas e laboratoriais não estão inclusas, esta nomenclatura é desaconselhada.

As variáveis observadas no exame histopatológico mostraram que muitas alterações não alcançam graus mais graves de lesão. Muitas lesões têm predomínio multifocal, o que talvez indique uma resposta homogênea do fígado, mesmo que isto não signifique maior agressão ao parênquima.

A natureza do infiltrado inflamatório é um referencial maior para a avaliação da agressão ao fígado, pois permite uma projeção sobre a persistência e a intensidade da lesão, o que fornece subsídios para o prognóstico. Embora Gayotto e Alves (1995) afirmem que plasmócitos não têm valor prognóstico em fase aguda de doença hepática, a presença de infiltrado linfoplasmocitário no fígado sempre é um indício de atividade inflamatória marcante no fígado e forte tendência à persistên-

\section{Referências}

ADAMUS, C.; DAUBIÉ-BUGGIN, M.; IZEMBART, A.; SONRIER-PIERRE C.; GUIGAND, L.; MASSON, M.T.; ANDRE-FONTAINE, G.; WYERS, M. Chronic hepatitis associated with lepstopiral infection in vaccinated beagles. J. Comp. Pathol., v. 117, p. 311-328, 1997.

ANDERSSON, M.; SEVELIUS, E. Breed, sex and age distribution in dogs with chronic liver disease: a demographic study. J. Small. Anim. Pract., v. 32, p. 1-5, 1991.

Circulating autoantibodies in dogs with chronic liver disease. J. Small. Anim. Pract., v. 33, p. 389-94, 1992.

BIANCHI, L. Liver biopsy interpretation in hepatitis. Part I. Presentation of critical morphologic features used in diagnosis (glossary). Pathol. Res. Pract., v. 178, n. 1, p. 2-19. 1983.

BISHOP, L.; STRANDBERG, J. D.; ADAMS, R. J.; BROWNSTEIN, D. G. PATTERSON, R. Chronic active hepatitis in dogs associated with Leptospires. Am. J. Vet. Res., v. 40, n. 6, p.839-844, 1979.

CENTER, S. A. Pathophysiology and laboratory diagnosis of liver disease. In: ETTINGER, S.J. (Ed.). Textbook of Veterinary Internal Medicine. Philadelphia: W.B. Saunders, 1995. v. 2, p. 1261-1312.

CENTER, S. A. Chronic liver disease in the dog: important concepts and management considerations. In: WALTHAM SYMPOSIUM LIVER DISEASE - PRACTICAL PERSPECTIVES, 1996, Birmingham, Proceedings... Birmingham: BSAVA, 1996, p. 5-7.

CHEVAUER,M:;GUERRE,S.;CHOSSEGROS, P;:GERARD, F;GRIMAUD, J. A. A histological semiquantitative scoring system for evaluation of hepatic fibrosis in needle liver biopsy specimens: comparison with morphometric studies. Hepatology, v. 20, p. 349-355, 1994.

DIAL, S. M. Clinicopathologic evaluation of the liver. Vet. Clin. North Am. Small Anim. Clin., v. 25, n. 2, p. 257-276, 1995.

DILLON, R. The liver in systemic disease: an innocent bystander. Vet. Clin. North Am. Small Anim. Clin., v. 15, p. 97-118, 1985.

FUENTEALBA, C.; GUEST, S.; HAYWOOD, S.; HORNEY, B. Chronic hepatitis: a restrospective study in 34 dogs. Can. Vet. J., v. 38, p. 365373, 1997.

GAYOTTO, L. C. C.; ALVES, V. A. F. Marcadores histopatológicos de doenças hepáticas. In: MATTOS, A. A. et al. (Ed.). Compêndio de Hepatologia. São Paulo: Fundo Editorial BYK, 1995, p. 57-76.

HARDY, R. M. Chronic hepatitis: an emerging syndrome in dogs. Vet. Clin. North Am. Small. Anim. Clin., v. 15, n. 1, p. 135-150, 1985.

ISHAK, K. G. Chronic hepatitis: morphology and nomenclature. Mod. Pathol., v. 7, n. 6, p. 690-713, 1994.

JARRETT, W. F. H.; O'NEIL, B. W.; LINDHOLM. Persistent hepatitis and chronic fibrosis induced by canine acidophil cell hepatitis virus. Vet. Rec., v. 120, p. 234-235, 1987. cia da lesão e evolução para cronicidade do processo, no que concordam Zawie e Gilbertson (1985).

A caracterização dos tipos de colágeno é condizente com os referidos por Sherlock (1989), Montes e Junqueira (1991) e Chevallier et al. (1994) e, embora não tenha permitido caracterizar fases de transição, evidencia que as hepatites crônicas dentro da sua história natural evoluem, ainda que não invariavelmente, para a cirrose. Segundo Johnson (1995), a cirrose alcança $15 \%$ de prevalência nas afecções hepáticas em cães.

A biópsia hepática é um recurso diagnóstico excelente para caracterização da doença hepática, fornecendo subsídios essenciais para instituição da terapia e para o seguimento do paciente. $E$ interessante que o diagnóstico morfológico consiga ser traduzido clinicamente e os critérios citohistológicos permitem uma avaliação precisa do estágio de evolução da doença hepática, norteando a conduta clínica.

JONES, T. C.; HUNT, R.D.; KING, N. W. The digestive system. In: Veterinary Pathology. Baltimore: Williams \& Wilkins, 1997.p.1043-1109. JOHNSON, S. E. Diseases of the liver. In: ETTINGER, S.J. (Ed.). Textbook of Veterinary Internal Medicine. Philadelphia: W.B. Saunders, 1995. v. 2, p.1313-1357.

KELLY, W. R. The liver and biliary system. In: JUBB, K.V.F. et al. (Ed.). Pathology of Domestic Animals. New York: Academic Press, 1993. v. 2, p. 319-406.

LEES, P.; MAY, S. A.; McKELLAR, Q.;A. Pharmacology and therapeutics of non-steroidal anti-inflammatory drugs in the dog and cat: general pharmacology. J. Small Anim. Pract., v. 32, p. 183-193, 1991.

LEVEILLE-WEBSTER, C. R.; CENTER, S. A. Chronichepatitis: therapeutic considerations. In: BONAGURA, J.D. (Ed.). Kirk's Current Veterinary Therapy XII Small Animal Practice. Philadelphia: W. B. Saunders, 1995. p. $749-756$.

LUDWIG, J. Histopathological diagnosis and terminology of chronic hepatitis. J. Hepatol., v. 23, supp. 1, p. 49-53, 1995.

MCKELLAR, Q. A.; MAY, S. A.; LEES, P. Pharmacology and therapeutics of non-steroidal anti-inflammatory drugs in the dog and cat: individual agents. J. Small Anim. Pract., v. 32, p. 225-235, 1991.

MONTES, G. S.; JUNQUEIRA, L. C. U. The use of the picrossirius polarization method for the study of the biopathology of collagen. Mem. Inst. Oswaldo Cruz, v. 96, supp. 111, p.1-11, 1991.

PESSAYRE, D. Role of reactive metabolites in drug-induced hepatitis. J. Hepatol., v. 23, supp. 1, p. 16-24, 1995.

POITOUT, F; WEISS, D. J.; ARMSTRONG, P. J. Cell-mediated immune responses to liver membrane protein in canine chronic hepatitis. Vet. Immunol. and Immunopathol., v. 57, n. 3/4, p. 169-178, 1997.

RAKISH, P. M.; PRASSE, K.; LUKERT, P.; CORNELIUS, L.M. Immunohistochemical detection of canine adenovirus in paraffin sections of liver. Vet. Pathol., v. 23, p.478-484, 1986.

RUTGERS, H. C.; HAYWOOD, S. Chronic hepatitis in the dog. J. Small Anim. Pract., v. 29, p. 679-690, 1988.

SARLI, G. Chronic active hepatitis in the dog. Veterinaria, v. 6, n. 1, p. 66-73, 1992

SEVELIUS, E. Diagnosis and prognosis of chronic hepatitis and cirrhosis in dogs. J. Small Anim. Pract., v. 36, p. 521-528, 1995.

SEVELIUS, E.; ANDERSSON, M.; JÖNSSON, L. Hepatic accumulation of alpha-1-antitypsin in chronic liver disease in the dog. J. Comp. Path., v. 111, p. 401-412, 1994.

SEVELIUS, E.; JÖNSSON, L. H. Pathogenic aspects of chronic liver disease in the dog. In: BONAGURA, J.D. (Ed.). Kirk's Current Veterinary Therapy XII Small Animal Practice. Philadelphia: W.B.Saunders, 1995. p. 740-742. 
SHERLOCK, S. Needle biopsy of the liver. In Diseases of the Liver and Biliary System. Oxford: Blackwell Scientific, 1989. p. 26-34.

STROMBECK, D. R.; MIUER, L. M.; HARROLD, D. Effects of corticosteroid treatment on survival time in dogs with chronic hepatitis: 151 cases (19771985). J. Am. Vet. Med. Assoc., v. 193, n. 9, p.1109-1113, 1988.

TESKE, E.;BRINKHUIS, B. G. A. M.;BODE, P.; VANdenINGH, T.S. G. A.M. ROTHUIZEN, J. Cytological detection of copper for the diagnosis of inhented copper toxicosis in Bedlington terriers. Vet. Rec., v. 131, p. 30-32, 1992. TWEDT, D. C. Cirrhosis: a consequence of chronic liver disease. Vet. Clin. North Am. Small Anim. Pract., v. 15, n. 1, p.151-176, 1985.

THORNBURG, L. P.; CHILDS, A.; TOOMEY, A. A.; ROUDEBUSH, P. Postnecrotic canine cirrhosis - 1. Clinicopathologic features. VM/SAC v. 78, p. 43-50, 1983a.

THORNBURG, L. P.; DANIELS, G. M. Postnecrotic canine cirrhosis, end-stage disease. VM/SAC, v. 78, p. 725-731, 1983b.
THORNBURG, L. P.; NELSON, S. L.; KINTNER, L. D. Postnecrotic canine cirrhosis - 2. Diagnosis, prognosis, and treatment. VM/SAC, v. 78, $p$. 168-173, 1983c.

THORNBURG, L. P.; TOOMEY, A. A.; LaBERGE, C. Postnecrotic cirmosis -3. Subacute disease. VM/SAC, v. 78, p.379-382, 1983d.

THORNBURG, L. P.; ROTTINGHAUS, G.; DENNIS, G.; CRAWFORD, S. The relationship between hepatic copper content and morphologic changes in the liver of West Highland White Terriers. Vet. Pathol., v. 33, p. 656-661, 1996.

WEISS, D. J.; ARMSTRONG, P. J.; MRUTHYUNJAYA, A: Anti-liver membrane protein antibodies in dogs with chronic hepatitis. J. Vet. Intern. Med., v. 9, n. 4, p. 267-271, 1995.

ZAWIE, D. A.; GILBERTSON, S. R. Interpretation of liver biopsy: A clinician's perspective. Vet. Clin. North Am. Small An. Clin., v. 15, n. 1,
p. 67-76, 1985 . 